\title{
BMJ Open Pre-post changes in main outcomes of medical rehabilitation in Germany: protocol of a systematic review and meta-analysis of individual participant and aggregated data
}

\author{
Michael Schuler, ${ }^{\oplus 1,2}$ Kathrin Murauer, ${ }^{1,2}$ Stephanie Stangl, ${ }^{2}$ Anna Grau, ${ }^{2}$ \\ Katharina Gabriel, ${ }^{2}$ Lauren Podger, ${ }^{3}$ Peter U Heuschmann, ${ }^{2,4}$ Hermann Faller ${ }^{1}$
}

To cite: Schuler M, Murauer K, Stangl S, et al. Pre-post changes in main outcomes of medical rehabilitation in Germany: protocol of a systematic review and metaanalysis of individual participant and aggregated data. BMJ Open 2019;9:e023826. doi:10.1136/ bmjopen-2018-023826

- Prepublication history and additional material for this paper are available online. To view these files, please visit the journal online (http://dx.doi org/10.1136/bmjopen-2017023826).

Received 25 April 2018 Revised 25 March 2019 Accepted 1 May 2019

Check for updates

(C) Author(s) (or their employer(s)) 2019. Re-use permitted under CC BY-NC. No commercial re-use. See rights and permissions. Published by BMJ.

For numbered affiliations see end of article.

Correspondence to Dr Michael Schuler; m.schuler@uni-wuerzburg.de

\section{ABSTRACT}

Introduction Multidisciplinary, complex rehabilitation interventions are an important part of the treatment of chronic diseases. However, little is known about the effectiveness of routine rehabilitation interventions within the German healthcare system. Due to the nature of the social insurance system in Germany, randomised controlled trials examining the effects of rehabilitation interventions are challenging to implement and scarcely accessible. Consequently, alternative pre-post designs can be employed to assess pre-post effects of medical rehabilitation programmes. We present a protocol of systematic review and meta-analysis methods to assess the pre-post effects of rehabilitation interventions in Germany.

Methods and analysis The respective study will be conducted within the Preferred Reporting Items for Systematic Reviews and Meta-Analysis guidelines. A systematic literature review will be conducted to identify studies reporting the pre-post effects (start of intervention vs end of intervention or later) in German healthcare. Studies investigating the following disease groups will be included: orthopaedics, rheumatology, oncology, pulmonology, cardiology, endocrinology, gastroenterology and psychosomatics. The primary outcomes of interest are physical/mental quality of life, physical functioning and social participation for all disease groups as well as pain (orthopaedic and rheumatologic patients only), blood pressure (cardiac patients only), asthma control (patients with asthma only), dyspnoea (patients with chronic obstructive pulmonary disease only) and depression/ anxiety (psychosomatic patients only). We will invite the principal investigators of the identified studies to provide additional individual patient data. We aim to perform the meta-analyses using individual patient data as well as aggregate data. We will examine the effects of both study-level and patient-level moderators by using a metaregression method.

Ethics and dissemination Only studies that have received institutional approval from an ethics committee and present anonymised individual patient data will be included in the meta-analysis. The results will be presented in a peer-reviewed publication and at research conferences. A declaration of no objection by the ethics
Strengths and limitations of this study

- This will be the first study to summarise pre-post research data on the effects of rehabilitation interventions in Germany in a range of different disease groups.

- We will perform meta-analyses using individual patient data and aggregate data.

- We will examine the effects of both patient-level moderators and study-level moderators.

- Causal interpretations of the pre-post effects will not be reported due to the inclusion of observational studies only.

- Analyses will be performed on studies investigating interventions lasting 3-4 weeks only, as provided routinely in Germany.

committee of the University of Würzburg is available (number 20180411 01).

Trial registration number CRD42018080316.

\section{INTRODUCTION}

Rehabilitation interventions are designed to reduce disability and improve functioning, and as such are regarded as key for optimising health in the 21 st century ${ }^{1}$ for people with chronic and acute conditions. The implementation of rehabilitation interventions varies vastly from country to country. ${ }^{2-5}$ In contrast to outpatient intervention programmes in other countries, in Germany, interventions are mostly conducted as 3-week or 4-week inpatient programmes in specialised rehabilitation centres. Rehabilitation is a part of the social insurance system, mostly provided by the German Statutory Pension Insurance (GPI), German statutory health insurances or private health insurances. For example, providing certain formal prerequisites are met, a patient is entitled to apply 
for medical rehabilitation at the GPI. If this application is confirmed by the GPI, the patient has the legal right to access a medical rehabilitation programme. The current funding system for German rehabilitation programmes impedes the use of randomised controlled trials (RCTs) to examine the effectiveness of the interventions (although there are a few exceptions ${ }^{6}$ ), as well as the comparison of results from RCTs across other healthcare systems.

To enhance rehabilitation research in Germany, in 1998 the GPI and the Federal Ministry of Education and Research funded the initiation of different rehabilitation research networks. In the following years, the number of rehabilitation research projects increased dramatically. This observed increase was also supported by additional funding initiatives. The research goals of these projects were broad and included the development, evaluation and implementation of intervention components, ${ }^{78}$ psychometric validation studies ${ }^{910}$ and prediction studies. ${ }^{11-13}$ Many of these studies used a variant of a pre-post design, with baseline assessments carried out at the start of the intervention and one or more postintervention assessments carried out at the end of rehabilitation or later, respectively. Previous literature reviews have summarised the overall findings across these pre-post studies. ${ }^{14-17}$ The conclusions drawn from these reviews helped to shape our current understanding of changes in important outcomes over the course of medical rehabilitation programmes. The previous literature highlights the importance of investigating the effects of multiple outcomes, as well as keeping in mind that the effects may differ dramatically across different outcomes. Some of these outcomes are generic, for example, quality of life, anxiety or depression, whereas other outcomes should be considered disease-specific, such as blood pressure for cardiac rehabilitation or pain for orthopaedic conditions.

The cited reviews only included studies that were published over a decade ago, the most recent studies investigating German rehabilitation programmes were not included. Additionally, in 1996 a systemic legal change resulted in a modification to the rehabilitation programme framework in German healthcare. The time spent in inpatient rehabilitation was reduced from 4 to 5 weeks to 3 weeks. The majority of previous reviews included studies from 1990 (or earlier) onwards and did not examine the length of hospital stay or study date as potential moderating factors. Hence, it is not clear whether the results of the cited studies can be used in contemporary research as unbiased estimates of the expected pre-post changes in outcomes of medical rehabilitation. Additionally, to date there have been no studies conducted on other important diagnostic groups like cancer or pulmonology diseases.

The present study aims to build on previous systematic literature reviews and expand the findings to include other disease groups such as orthopaedics, rheumatology, cardiology, pulmonology, oncology, endocrinology, gastroenterology and psychosomatics. Furthermore, this study will not solely rely on aggregated data $(\mathrm{AD})$ as done in previous reviews, but will also include individual patient data (IPD). ${ }^{18}$ Meta-analyses of IPD are regarded as 'gold standard' as they have many advantages over meta-analyses based on aggregated data. The main advantage of IPD is that the statistical analyses can be tailored to appropriately accommodate the features of the data and the differences in study design, for example, study-specific differences in the handling of missing data or study-specific approaches to measure effect size. Moreover, analyses of individual moderator variables such as sex, age or disease severity can be carried out.

\section{Objectives of the study}

The primary objectives of this study are as follows:

1. To identify the overall pre-post effects on important generic outcomes using values measured at the start of a medical rehabilitation intervention and at the end (or within $3, \leq 6, \leq 12$ and $>12$ months following completion of the intervention).

2. To identify disease-specific pre-post effects on important generic and disease-specific outcomes at the start of a medical rehabilitation intervention and at the end (or within $3, \leq 6, \leq 12$ and $>12$ months following completion of the intervention).

(For a detailed description of the outcomes, see below). Furthermore, the following secondary aims and objectives will be addressed:

3. To identify patient-level (age, sex, severity of disease) and study-level variables (year of study, goal of study, number of research groups) that may moderate the effects as delineated in the primary research questions.

4. To summarise the prominent research questions examined in German rehabilitation research since 1998 with studies using pre-post designs.

\section{METHODS AND ANALYSES}

The protocol was designed in accordance with the Preferred Reporting Items for Systematic Reviews and Meta-Analyses for Protocols (PRISMA-P) 2015 guideline, ${ }^{19}$ as well as the Preferred Reporting Items for Systematic Reviews and Meta-Analyses of Individual Participant Data (PRISMA-IPD) guideline. ${ }^{20}$ The protocol is registered on the PROSPERO database (trial number: CRD42018080316).

\section{Eligibility criteria}

Types of studies

We will include literature published in English and German languages only. Studies will be included if the patient recruitment was conducted from 1998 onwards and if the patient data were collected prospectively. Retrospective publications of routine data from a clinic will not be included. Furthermore, studies examining routine data for insurance agencies will not be included in the analyses. Primary studies will only be included within IPD meta-analysis if ethical approval is confirmed.

A limited number of published studies have investigated the pre-post effects of medical rehabilitation 
Table 1 Disease groups and the most frequently reported diseases and/or health conditions

\begin{tabular}{|c|c|}
\hline Disease group & $\begin{array}{l}\text { Frequently reported diseases and/or } \\
\text { health conditions }\end{array}$ \\
\hline Orthopaedics & Chronic low back pain, osteoarthritis \\
\hline Rheumatology & $\begin{array}{l}\text { Rheumatoid arthritis, ankylosing } \\
\text { spondylitis }\end{array}$ \\
\hline Cardiology & $\begin{array}{l}\text { Chronic heart failure, coronary artery } \\
\text { disease }\end{array}$ \\
\hline Oncology & Breast cancer, prostate cancer \\
\hline Pulmonology & $\begin{array}{l}\text { Chronic obstructive pulmonary disease, } \\
\text { asthma }\end{array}$ \\
\hline Endocrinology & Diabetes mellitus \\
\hline Gastroenterology & Inflammatory bowel disease \\
\hline Psychosomatics & Depression disorder, anxiety disorder \\
\hline
\end{tabular}

in Germany. However, variations of a pre-post design have been adopted in previous studies to address other research questions, for example, testing efficacy of novel intervention components or to examine the psychometric properties of a questionnaire. Therefore, we will include studies addressing a range of primary research questions. All included studies must fulfil the following inclusion criteria:

- Studies will use a pre-post design to investigate medical rehabilitation in Germany.

- The measurement of preintervention values will be carried out at baseline or up to 4 weeks prior to the intervention.

- The measurement of postintervention values will be carried out at: end of the intervention, $\leq 3, \leq 6, \leq 12$ and $>12$ months.

\section{Population}

We will restrict our overview of studies to subjects aged 18 years or older, and who have received medical rehabilitation in Germany for any of the following disease groups: orthopaedics, rheumatology, cardiology, pulmonology, oncology, endocrinology, gastroenterology and psychosomatics (table 1). Additionally, table 1 lists the diseases and/or health conditions within each disease group that patients most frequently receive rehabilitation for in Germany.

We will exclude studies of subjects who have received medical rehabilitation for neurological disorders (ie, traumatic brain injury, stroke and neural infections) and addiction. In comparison to rehabilitation programmes for other health conditions (table 1), the interventions designed for neurological disorders and addictions can vary in duration. Furthermore, self-reported accounts from patients receiving neurological rehabilitation can be impacted by recall bias. Patients with traumatic brain injury or stroke can often overestimate their abilities and the status of their health conditions at the start of the intervention, leading to biased pre-post changes. ${ }^{21}$

\section{Interventions}

We will restrict our overview to studies that examine medical rehabilitation interventions in Germany only. The studies will include either inpatient or full-time outpatient interventions carried out for a minimum of 21 days. Studies will be included irrespective of the type (health or pension) of insurance agency that provides the funding for the programme.

\section{Comparisons}

The intervention effect will be calculated as the subtraction of patient's postintervention values from their baseline values. An untreated control group will not be used in this study.

\section{Types of outcome measures}

We will include studies that report preintervention and postintervention data for one or more of the outcomes presented in table 2.

Return to work (after 3 months) will be assessed as a primary outcome for all disease groups when subjects are of working age (18-65 years). Return to work will be operationalised as either a dichotomous variable (yes/

\section{Table 2 Primary and secondary outcomes}

\begin{tabular}{ll}
\hline Primary outcomes & Secondary outcomes \\
Physical and mental quality of life/subjective health* & Depression and anxiety symptomst \\
Physical functioning* & Self-management* \\
Social participation* & Coping with pain (orthopaedic and rheumatologic patients \\
Pain (orthopaedic and rheumatologic patients only) & only) \\
Blood pressure (cardiac patients only) & Fear of progression (patients with cancer only) \\
Asthma control (patients with asthma only) & Lung function (patients with asthma and COPD only) \\
Dyspnoea (patients with COPD only) & Functional capacity (patients with COPD only) \\
Depression and anxiety symptoms (psychosomatic & disease only) \\
rehabilitation patients only) & Subjective work ability* \\
\hline
\end{tabular}

${ }^{*}$ Outcomes will be collected for all disease groups.

†Outcome will be collected for all disease groups except for 'psychosomatic rehabilitation'.

COPD, chronic obstructive pulmonary disease; HbA1c, haemoglobin A1c. 
Table 3 Study-level and patient-level moderator variables

\begin{tabular}{ll} 
Study-level moderators & Patient-level moderators \\
\hline Control group study (yes/no) & Sex \\
Number of patients & Age \\
Publication year (of an article) & Follow-up treatment after acute care hospital stay \\
Start of project (year) & ('Anschlussheilbehandlung') vs rehabilitation because of \\
Approval by an ethics committee as mentioned in the & chronicity ('Heilverfahren') \\
publication (aggregate data only; yes/no) & Sick leave immediately preceding inpatient rehabilitation \\
Methodological quality of study & (yes/no and/or days of sick leave) \\
Published in an impact factor journal (at time of publication) & Symptom burden (eg, GOLD-Stage in patients with COPD, \\
& NYHA class in patients with chronic heart failure) \\
& Baseline value in respective outcome
\end{tabular}

COPD, chronic obstructive pulmonary disease; GOLD, Global Initiative of Lung Disease; NYHA, New York Heart Association.

no) or as a discrete variable (the number of days a subject is unable to work following the intervention).

The remaining outcomes will be assessed using studies that include psychometrically validated instruments, for example, 36-Item Short Form Survey ${ }^{22}$, Hospital Anxiety and Depression Scale ${ }^{23}$ or Asthma Control Test. ${ }^{24}$ Outcomes assessed with an instrument that was developed in the respective study will not be included.

\section{Moderator variables}

We will examine the study-level and patient-level moderator variables presented in table 3 .

\section{Data collection}

Methods for the identification and selection of studies

Several sources of information will be used to identify the studies meeting the inclusion criteria. First, we will undertake a comprehensive search for published literature in the following electronic databases: MEDLINE (via PubMed), PsycINFO, Psyndex and LIVIVO. Searches for any relevant literature will be carried out by free text combined with Boolean operators. The search strategies will be adapted accordingly for each of the electronic databases (see online supplementary additional file 1). Only studies published between 1 January 1998 and 9 August 2017 will be considered. Second, the web pages of identified rehabilitation research funders in Germany, as well as the organisations supporting research projects, will be screened for studies that fulfil our eligibility criteria (for complete list, see online supplementary additional file 1). Third, conference transcripts, the German Registry of Clinical Trials (DRKS) and the reference lists of all the relevant and included publications will be searched for additional studies.

\section{Electronic databases}

The results from the literature search will be downloaded and saved into the Citavi reference management software ${ }^{25}$ Following the initial identification and removal of duplicates, an independent rater will screen the titles and abstracts against the eligibility criteria. Articles that clearly do not meet the inclusion criteria (published guidelines, studies with children, qualitative studies, reviews, etc) will be excluded. During this step 10\% of the identified articles will be concurrently selected to be assessed for eligibility by two independent raters. For the remaining articles, the full-texts will be obtained and screened for eligibility by two independent raters. Throughout all of the steps, any inter-rater disagreements will be resolved by discussion or consultation with a third independent investigator. The study selection process will be summarised in the final report using a PRISMA flow diagram, ${ }^{26}$ along with the rationale for excluding articles during the fulltext screening. The study selection processes for IPD and $\mathrm{AD}$ will be presented separately.

\section{Data extraction and management}

Two independent raters will extract data from the included publications. Additionally, we will contact the authors of all eligible studies to request the original datasets and confirmation of ethical approval. We will take several steps to ensure that investigators demonstrate compliance with regulations when requesting access to original data. First, we will contact (via telephone and email if possible) the primary study authors. Second, we will draft a written agreement that states the data will only be used to answer the respective research questions. Third, we will provide regular project progress updates to all the authors who supply original data sets. Fourth, on completion of the project the data provider will be invited to review and provide suggestions on how the combined data set (or parts of it) can be used to examine further research questions (by either our research group or other researchers). The data that will be extracted for all studies meeting the inclusion criteria is presented in table 4 .

\section{Dealing with studies with two or more treatment arms}

We anticipate that some of the included clinical trial studies will have a multiarm design (two or more treatment arms). We are primarily interested in the mean pre-post intervention effects, as such we will only extract (AD analyses) or compute (IPD analyses) the results of the combined study arms. We will compute combined parameters (ie, weighted means) for IPD data if the results are reported separately for each of the study arms. 
Table 4 Study-level data and patient-level data that will be extracted

\begin{tabular}{|c|c|}
\hline Study-level data & Patient-level data \\
\hline $\begin{array}{l}\text { Names and affiliations of study coordinators } \\
\text { Start date of the project } \\
\text { Project ID } \\
\text { Aim of project, primary and secondary outcomes } \\
\text { - Typessment instruments } \\
\text { study with more than one group, psychometric validation } \\
\text { study, other } \\
\text { - Measurement time periods } \\
\text { - Participants: selection, inclusion and exclusion criteria } \\
\text { - Study protocol (if available) } \\
\text { - Complete list of all publications from these data } \\
\text { - Source of funding } \\
\text { - Numbebook (for available original data sets only) } \\
\text { Number of dropouts }\end{array}$ & $\begin{array}{l}\text { Demographics: age, sex, education, employment status } \\
\text { Pre-test and post-test values of the respective outcomes (item } \\
\text { level, if available) } \\
\text { Time of measurement } \\
\text { Rehabilitation with stable patients ('Heilverfahren') or } \\
\text { rehabilitation with patients after acute care hospital stay } \\
\text { ('Anschlussheilbehandlung'), eg, because of a myocardial } \\
\text { infarction or chronic obstructive pulmonary disease } \\
\text { exacerbation } \\
\text { Symptom burden }\end{array}$ \\
\hline
\end{tabular}

In a recent study, Huh et $a l^{27}$ presented a statistical model that can include multiple intervention arms per study without collapsing the data across the intervention arms. We aim to adopt this approach as part of our sensitivity analyses and will discuss any differences when compared with the primary analysis method.

\section{Data management of aggregate data}

AD from all included studies will be extracted and collated in an electronic database by one investigator. Validation of the entered data will be carried out by a second investigator. Any disagreement will be discussed and resolved by a third independent investigator.

\section{Data management of individual patient data}

IPD from all included studies will be transferred electronically and collated. Following a consultation with the authors of the included studies and the data protection officer of the University of Würzburg, the de-identification of data will be carried out prior to data transfer (eg, name, address and date of birth). Additionally, a copy of every transferred data set containing anonymised information will be archived prior to data analysis. Access to these data will be restricted to specific staff members and permission-dependent user logins. The included studies will be separately reviewed to confirm internal plausibility and consistency with the published data. The plausibility checks will include error checks on the measured variables, screening for outliers or duplicates and a comparison of the IPD results with the published results. The original authors will be contacted to clarify outliers or unusual values, or to discuss any discrepancies between our findings and the published results. Following the completion of the quality check, all data sets will be merged into one and a unique identification number will be generated for each participant within the new joint data set. Each variable within the joint data set will be described in a codebook.
Assessing the quality of included studies

We will critically appraise the included studies, focusing on a comparison of the study quality. The risk of bias will be assessed using a modified version of the ROBINS-I risk of bias tool. ${ }^{28} \mathrm{~A}$ second rater will provide an additional validation of the bias assessments for $20 \%$ of the included studies. Any disagreements will be resolved by discussion or a third person. The full appraisal results will be included in the final review along with a summary of the quality assessment within the narrative synthesis. The quality scores for each of the included studies will be presented in the evidence tables.

\section{Data synthesis}

Missing data

We anticipate that missing data such as mean values, SEs/ SDs or moderator variables may occur. For AD analyses, we will use the following strategy to address missing data: first, we will attempt to compute the missing values based on all suitable available data. Second, any remaining missing data will be addressed via multiple imputation methods. We will create 10 imputed data sets and report the pooled results. ${ }^{29}$ Third, we will conduct a sensitivity analysis using all available data only. The proportion of missing data identified for the included studies and the results of the sensitivity analyses will reported.

For IPD analysis, the method of multiple imputation will be employed to address the missing data values. ${ }^{29} \mathrm{We}$ will report the proportion of the identified missing data and summarise the possible explanations for any missing data.

For both $\mathrm{AD}$ and IPD analyses, imputation of missing data will only be carried out if the data were unavailable for the following reasons: (1) the data are not reported (AD analyses only) and (2) the data for specific questionnaire items or a given time point is not reported (IPD analyses only). Data that are not assessed in the respective study will not be imputed. 


\section{Statistical analysis}

To address the first research question, we intend to employ three different meta-analytic methods: 1) $\mathrm{AD}$ analyses, including all studies using published aggregate data, 2) two-stage IPD analyses, including IPD if available and $\mathrm{AD}$ if IPD is unavailable and 3) one-stage IPD analyses, including all studies using available IPD. ${ }^{18}$ One-stage IPD analysis can only be conducted across studies that use the same outcome measure. The analysis of studies with different outcome measures will be conducted using a two-stage approach. ${ }^{30}$ To address the second research question, we will conduct the above-mentioned types of meta-analyses for each disease group separately. The meta-analyses will use a random-effects model to generate summary estimates of effect. Continuous outcomes will be summarised using the standardised effect size (SES, with $95 \% \mathrm{CI}){ }^{31}$ The SES will be calculated as the mean difference in values prerehabilitation and postrehabilitation divided by the SD of the assessments at the start of the intervention. Dichotomous outcomes (return to work) will be summarised using the weighted risk ratio (with 95\% CI). Heterogeneity will be assessed using the $\mathrm{I}^{2}$ statistic. An $\mathrm{I}^{2}$ value of $25 \%$ is considered to represent low heterogeneity, $50 \%$ is considered moderate and $75 \%$ is considered high. ${ }^{32}$

To address the third research question, we intend to use mixed-effects meta-regressions to examine IPD subjectlevel moderators. ${ }^{33}$ We will adopt a one-stage or two-stage analysis approach depending on the variation of outcome measures reported in the included studies.

We will employ a two-stage IPD analysis approach, in conjunction with assessment of $\mathrm{AD}$, to examine studylevel moderators. Each moderator will be tested separately, however combinations of two or more moderators could be tested depending on the number of available studies.

The relevant data extracted from all eligible studies will be presented in the evidence tables.

\section{Additional analyses}

\section{Meta-bias}

To explore any instances of reporting bias we will examine all available protocols for the included studies that were published prior to completion of recruitment for the respective study. The German Clinical Trial Register (https://www.drks.de/drks_web/) will be screened to access the study protocols and to examine the presence of selective reporting of outcomes. Furthermore, funnel plots will be used to assess publication bias. ${ }^{34}$

\section{Confidence in cumulative evidence}

The quality of the cumulative results will be discussed using the criteria presented by the Grading of Recommendation Assessment, Development and Evaluation working group. ${ }^{35}$ This includes the risk of bias, consistency, directness, precision and publication bias. However, our analyses will only include observational studies, as such investigating and reporting the causal interpretations of the pre-post effects is not necessary. For this reason, the recommended scheme to classify the Quality of a body of evidence ${ }^{36}$ would not be very informative and thus will not be included in our study. Rather, we will present a detailed discussion of the quality criteria as stated above.

\section{Patient and public involvement}

Patients were not involved throughout the development of the research questions and the study design. Our findings may inform patients about realistic postrehabilitation intervention changes in a variety of important and meaningful patient-related outcomes.

\section{DISCUSSION}

The respective study will provide preintervention and postintervention estimates of change in key patient-related rehabilitation outcomes. We will focus our investigation on the rehabilitation interventions aimed at the most significant and frequently reported medical conditions in Germany. To our knowledge, this will be the first study that systematically investigates the potential effects of both patient-level and study-level moderators on pre-post changes following rehabilitation in Germany. The results will be presented for each of the different time points (post-test and follow-up) to estimate the disease trajectories on completion of the programme. Additionally, we will aim to present the results for each disease group separately as well as overall (for available outcomes).

It will be potentially challenging to interpret pre-andpost changes in different disease groups as patients may have different baseline values and vary in the likelihood of improvement. For example, improvements in physical function may be more difficult to achieve in patients with chronically progressive disorders such as COPD in comparison to patients with other chronic illnesses like asthma. However, change in psychosomatic outcomes such as mental quality of life, depression and anxiety is perhaps less likely to be influenced by the nature of the underlying disease itself. Furthermore, the majority of patients receiving rehabilitation report a variety of comorbidities. ${ }^{37}$ Additionally, the rehabilitation interventions developed for different disease groups share many treatment components (eg, exercise and patient education) and have similar aims, that is, improvement of quality of life and social participation. As such, patients across different disease groups may share commonalities.

The findings from our study will build on the previous reviews of medical rehabilitation outcomes. For example, Mittag et $a l^{14}$ summarised findings from a cardiac rehabilitation programme and reported high pre-post effects in functional capacity ( $\mathrm{ES}=0.94)$, medium-sized effects in blood pressure as well as small effects in depression and anxiety. Hüppe and Raspe ${ }^{1516}$ examined pre-post design studies investigating changes in outcomes for patients with chronic back pain. The authors presented mediumto-high changes in vitality, depression and pain intensity and low-to-medium effects in physical functioning. 
Furthermore, Löschmann et al $l^{17}$ examined the pre-post effects of inpatient rehabilitation interventions for patients with psychosomatic disorders. The authors found a medium effect size $(\mathrm{ES}=0.51)$ overall.

However, it is important to note that the above findings were based on data published before 2005. Consequently, recent studies investigating rehabilitation interventions in Germany were not included in the above reviews.

One potential limitation of our study is an inability to interpret the results as a causal effect of the rehabilitation interventions. The influence of a variety of confounders on the results, such as regression to the mean or natural course of disease, cannot be ruled out, owing to the absence of a control condition. Alternatively, the results can be interpreted as the expected changes in patients' health status during a rehabilitation intervention in Germany, regardless of the mechanisms that caused these changes.

Taking these limitations into account, the results may supply health professionals, scientists and healthcare providers (HCPs) with a greater understanding of realistic and expected rehabilitation effects. The findings may prompt the improvement of medical rehabilitation interventions in Germany and provide a foundation when planning and interpreting future studies. For example, our results may be used to give patients representative information about the magnitude of pre-post changes following inpatient rehabilitation for a variety of important patient-related outcomes. Moreover, a better understanding of any significant study-level moderator may help HCPs identify patient groups experiencing minor changes as compared with other patient groups. Interventions aimed at the specific needs of these patient groups may be developed in the future. Also, scientists could use findings from the respective study to estimate the expected effects during medical rehabilitation. For example, if a scientist plans to test a new intervention component, our results could be used to estimate the hypothesised pre-post changes for a variety of outcomes for a control group.

In addition, it will be possible to use our findings to evaluate whether effect sizes in future studies should be considered as high, medium or low when compared with the previous findings. Furthermore, our results could be compared with the findings from international rehabilitation studies. In contrast to Germany, many countries provide outpatient rehabilitation interventions only. Our study may be used to compare the observed intervention effects across countries and healthcare systems, as well as any differences between inpatient and outpatient interventions. In summary, we anticipate the findings from our respective study will be of valuable to future research and help guide future practice in medical rehabilitation.

\section{Ethics and dissemination}

This paper contains the original study protocol. Any substantial modifications to the protocol will be noted according to the PRISMA-P 2015 guideline. These amendments will be documented in detail in the PROSPERO Register and will be described transparently.

\section{Author affiliations}

${ }^{1}$ Department of Medical Psychology and Psychotherapy, Medical Sociology and Rehabilitation Sciences, Julius-Maximilians-Universität Würzburg, Würzburg, Bayern, Germany

${ }^{2}$ Institute for Clinical Epidemiology and Biometry (ICE-B), Julius-MaximiliansUniversität Würzburg, Würzburg, Bayern, Germany

${ }^{3}$ ICON PIc, London, UK

${ }^{4}$ Comprehensive Heart Failure Center Würzburg, Julius-Maximilians-Universität Würzburg, Würzburg, Bayern, Germany

Correction notice This article has been corrected since it was published online. Author name Stefanie Stangl has been updated to Stephanie Stangl.

Acknowledgements The authors would like to thank Tara Petzke for proofreading the manuscript.

Contributors MS is the guarantor. MS and HF developed the study design. MS, HF, $\mathrm{PUH}, \mathrm{SS}, \mathrm{AG}, \mathrm{KM}$ and $\mathrm{KG}$ contributed to drafting the first version of the manuscript. $\mathrm{LP}$ has revised the entire manuscript linguistically. MS, HF and LP drafted the final version of the manuscript. All authors read and approved the final version of the manuscript for submission. All authors fulfill the authorship criteria of the International Committee of Medical Journal Editors.

Funding The study is funded by the German Statutory Pension Insurance (Deutsche Rentenversicherung Bund), Hohenzollerndamm 46/47, 10713 Berlin, Germany (grant number: 0423/40-64-50-59). Funding covers personnel, material and travelling expenses. This publication was funded by the German Research Foundation (DFG) and the University of Wuerzburg in the funding programme Open Access Publishing.

Competing interests None declared.

Ethics approval As this is a protocol for a systematic review, a formal ethics committee review is not required. A declaration of no objection by the ethics committee of the University of Würzburg is available (number 20180411 01).

Provenance and peer review Not commissioned; externally peer reviewed.

Open access This is an open access article distributed in accordance with the Creative Commons Attribution Non Commercial (CC BY-NC 4.0) license, which permits others to distribute, remix, adapt, build upon this work non-commercially, and license their derivative works on different terms, provided the original work is properly cited, appropriate credit is given, any changes made indicated, and the use is non-commercial. See: http://creativecommons.org/licenses/by-nc/4.0/.

\section{REFERENCES}

1. WHO. Rehabilitation 2030: a call for action., 2017. Meeting report. http://www.who.int/disabilities/care/rehab-2030/en/.

2. Bjarnason-Wehrens B, McGee H, Zwisler AD, et al. Cardiac rehabilitation in europe: Results from the european cardiac rehabilitation inventory survey. Eur J Cardiovasc Prev Rehabil 2010;17:410-8.

3. Grotle M, Klokkerud M, Kjeken I, et al. What's in the black box of arthritis rehabilitation? A comparison of rehabilitation practice for patients with inflammatory arthritis in northern Europe. $J$ Rehabil Med 2013;45:458-66.

4. Spruit MA, Pitta F, Garvey C, et al. Differences in content and organisational aspects of pulmonary rehabilitation programmes. Eur Respir J 2014;43:1326-37.

5. Mittag O, Welti F. [Comparison of medical rehabilitation in various European countries and the impact of European law on rehabilitation practice in Germany]. Bundesgesundheitsblatt Gesundheitsforschung Gesundheitsschutz 2017;60:378-85.

6. Schultz K, Seidl H, Jelusic D, et al. Effectiveness of pulmonary rehabilitation for patients with asthma: study protocol of a randomized controlled trial (EPRA). BMC Pulm Med 2017;17:49.

7. Meng K, Musekamp G, Seekatz B, et al. Evaluation of a selfmanagement patient education program for patients with chronic heart failure undergoing inpatient cardiac rehabilitation: study protocol of a cluster randomized controlled trial. BMC Cardiovasc Disord 2013;13:60

8. Bethge M, Herbold D, Trowitzsch L, et al. Work status and healthrelated quality of life following multimodal work hardening: a cluster randomised trial. J Back Musculoskelet Rehabil 2011;24:161-72. 
9. Schuler M, Musekamp G, Faller H, et al. Assessment of proximal outcomes of self-management programs: translation and psychometric evaluation of a German version of the Health Education Impact Questionnaire (heiQTM). Qual Life Res 2013;22:1391-403.

10. Farin E, Gramm L, Schmidt E. Taking into account patients' communication preferences: instrument development and results in chronic back pain patients. Patient Educ Couns 2012;86.

11. Streibelt M, Bethge M, Gross T, et al. Predictive validity of a screening instrument for the risk of non-return to work in patients with internal diseases. Arch Phys Med Rehabil 2017;98:989-96.

12. Mosshammer D, Ring C, Kaluscha R, et al. [Prediction of premature pension after stationary rehabilitation due to adipositas-a prognostic model based on routinely collected data of the State Insurance Institution of Baden-Württemberg]. Rehabilitation 2005;44:353-60.

13. Muche R, Rösch M, Flierl S, et al. [Development and validation of a prognosis model for predicting work capacity after rehabilitation based on routinely collected parameters]. Rehabilitation 2000;39:262-7.

14. Mittag O, Schramm S, Böhmen S, et al. Medium-term effects of cardiac rehabilitation in Germany: systematic review and metaanalysis of results from national and international trials. Eur $J$ Cardiovasc Prev Rehabil 2011;18:587-693.

15. Hüppe A, Raspe H. [Efficacy of inpatient rehabilitation for chronic back pain in Germany: update of a systematic review]. Rehabilitation 2005;44:24-33.

16. Hüppe A, Raspe H. [Efficacy of inpatient rehabilitation for chronic back pain in Germany: a systematic review 1980-2001] Rehabilitation 2003;42:143-54.

17. Löschmann C, Steffanowski A, Wittmann WW, et al. Meta-analysis of the effects of stationary pscychosomatic rehabilitation - MESTAstudy. Psychotherapie Psychosomatik Medizinische Psychologie 2005;55:105-05.

18. Riley RD, Lambert PC, Abo-Zaid G. Meta-analysis of individual participant data: rationale, conduct, and reporting. BMJ 2010;340:c221.

19. Shamseer L, Moher D, Clarke M, et al. Preferred reporting items for systematic review and meta-analysis protocols (PRISMA-P) 2015: elaboration and explanation. BMJ 2015;350:g7647.

20. Stewart LA, Clarke M, Rovers M, et al. Preferred Reporting Items for Systematic Review and Meta-Analyses of individual participant data: the PRISMA-IPD Statement. JAMA 2015;313:1657-65.

21. Menzel-Begemann A. Berufliche Orientierung in der medizinischen Neurorehabilitation. Weinheim: Beltz Juventa, 2012.
22. Bullinger M. German translation and psychometric testing of the SF-36 Health Survey: Preliminary results from the IQOLA project. Soc Sci Med 1995;41:1359-66.

23. Hermann-Lingen C, Buss U, Snaith R, et al; Depression Scale: Deutsche Version. Bern: Huber, 2005.

24. Nathan RA, Sorkness CA, Kosinski M, et al. Development of the asthma control test: a survey for assessing asthma control. J Allergy Clin Immunol 2004:113:59-65.

25. Citavi 5 [program]. 2017.

26. Moher D, Shamseer L, Clarke M, et al. Preferred reporting items for systematic review and meta-analysis protocols (PRISMA-P) 2015 statement. Syst Rev 2015;4:1.

27. Huh D, Mun EY, Larimer ME, et al. Brief motivational interventions for college student drinking may not be as powerful as we think: an individual participant-level data meta-analysis. Alcohol Clin Exp Res 2015;39:919-31.

28. Sterne JA, Hernán MA, Reeves BC, et al. ROBINS-I: a tool for assessing risk of bias in non-randomised studies of interventions. BMJ 2016;355:i4919.

29. Kunkel D, Kaizar EE. A comparison of existing methods for multiple imputation in individual participant data meta-analysis. Stat Med 2017;36:3507-32.

30. Riley RD, Lambert PC, Staessen JA, et al. Meta-analysis of continuous outcomes combining individual patient data and aggregate data. Stat Med 2008;27:1870-93.

31. Kazis LE, Anderson JJ, Meenan RF. Effect sizes for interpreting changes in health status. Med Care 1989;27(3 Suppl):S178-S189.

32. Higgins JP, Thompson SG, Deeks JJ, et al. Measuring inconsistency in meta-analyses. BMJ 2003;327:557-60.

33. Viechtbauer W. Accounting for Heterogeneity via Random-Effects Models and Moderator Analyses in Meta-Analysis. Journal of Psychology 2007;215:104-21.

34. Borenstein M, Hedges LV, Higgins JPT, et al. Introduction to MetaAnalysis. New York, NY: John Wiley \& Sons, 2009.

35. Guyatt $\mathrm{GH}$, Oxman AD, Schünemann HJ, et al. GRADE guidelines: a new series of articles in the Journal of Clinical Epidemiology. J Clin Epidemiol 2011;64:380-2.

36. Balshem $\mathrm{H}$, Helfand $\mathrm{M}$, Schünemann HJ, et al. GRADE guidelines: 3 . Rating the quality of evidence. J Clin Epidemiol 2011;64:401-6.

37. Vanfleteren LE. Does COPD stand for "COmorbidity with Pulmonary Disease"? Eur Respir J 2015;45:14-17. 